OPEN ACCESS

Edited by:

Kai J. Miller,

Mayo Clinic, United States

Reviewed by:

Burak Güçlü,

Boğaziçi University, Turkey

Sean Kevin Meehan,

University of Waterloo, Canada

*Correspondence:

Daniel R. Kramer

dankramer29@gmail.com

Specialty section:

This article was submitted to

Neuroprosthetics,

a section of the journal

Frontiers in Neuroscience

Received: 31 January 2019

Accepted: 25 July 2019

Published: 07 August 2019

Citation:

Kramer DR, Lamorie-Foote $K$, Barbaro M, Lee M, Peng T, Gogia A,

Liu CY, Kellis SS and Lee B (2019)

Functional Frequency Discrimination

From Cortical Somatosensory

Stimulation in Humans.

Front. Neurosci. 13:832.

doi: 10.3389/fnins.2019.00832

\section{Functional Frequency Discrimination From Cortical Somatosensory Stimulation in Humans}

\author{
Daniel R. Kramer ${ }^{1,2 *}$, Krista Lamorie-Foote ${ }^{3}$, Michael Barbaro ${ }^{3}$, Morgan Lee ${ }^{3}$, \\ Terrance Peng ${ }^{3}$, Angad Gogia ${ }^{3}$, Charles Y. Liu ${ }^{1,2}$, Spencer S. Kellis ${ }^{2,4,5}$ and Brian Lee ${ }^{1,2}$ \\ ${ }^{1}$ Department of Neurosurgery, University of Southern California, Los Angeles, CA, United States, ${ }^{2}$ Neurorestoration Center, \\ University of Southern California, Los Angeles, CA, United States, ${ }^{3}$ Keck School of Medicine, University of Southern \\ California, Los Angeles, CA, United States, ${ }^{4}$ Department of Biology and Biological Engineering, California Institute \\ of Technology, Pasadena, CA, United States, ${ }^{5}$ Tianqiao and Chrissy Chen Brain-Machine Interface Center, California \\ Institute of Technology, Pasadena, CA, United States
}

Recently, efforts to produce artificial sensation through cortical stimulation of primary somatosensory cortex (PSC) in humans have proven safe and reliable. Changes in stimulation parameters like frequency and amplitude have been shown to elicit different percepts, but without clearly defined psychometric profiles. This study investigates the functionally useful limits of frequency changes on the percepts felt by three epilepsy patients with subdural electrocorticography (ECoG) grids. Subjects performing a hidden target task were stimulated with parameters of constant amplitude, pulse-width, and pulse-duration, and a randomly selected set of two frequencies $(20,30,40,50,60$, and $100 \mathrm{~Hz}$ ). They were asked to decide which target had the "higher" frequency. Objectively, an increase in frequency differences was associated with an increase in perceived intensity. Reliable detection of stimulation occurred at and above $40 \mathrm{~Hz}$ with a lower limit of detection around $20 \mathrm{~Hz}$ and a just-noticeable difference estimated at less than $10 \mathrm{~Hz}$. These findings suggest that frequency can be used as a reliable, adjustable parameter and may be useful in establishing settings and thresholds of functionality in future $\mathrm{BCl}$ systems.

\footnotetext{
Keywords: somatosensation, cortical stimulation, brain computer interface, brain machine interface, sensory feedback control, electrocorticography, frequency
}

\section{INTRODUCTION}

For the millions of patients with somatosensory deficits from stroke, paralysis, or limb-loss, restoration of function has vast implications for health, and independence recovery. Somatosensory brain-computer interface $(\mathrm{BCI})$ presents a means to restore function in such individuals, where somatosensory input can potentially improve motor BCI (Andersen et al., 2004, 2010; Suminski et al., 2010; Fifer et al., 2012; Chestek et al., 2013; Lee et al., 2013; Aflalo et al., 2015; Bundy et al., 2016; Flesher et al., 2016; Hollins and Risner, 2016; Armenta Salas et al., 2018), or restore basic functions like bladder control. Although stimulation of peripheral nerves can potentially reproduce somatosensation (Raspopovic et al., 2014; Tan et al., 2014), to fully restore function in stroke patients or paralyzed individuals, cortical stimulation would be required. However, somatosensory BCI is at an early stage with limited work establishing the basic utility (Flesher et al., 2016; 
Armenta Salas et al., 2018; Lee et al., 2018), functionality (Baumgartner et al., 1991; Tan et al., 2014; Collins et al., 2017), and modalities (Raspopovic et al., 2014; Tan et al., 2014; Vidal et al., 2016; Collins et al., 2017; Armenta Salas et al., 2018; Lee et al., 2018).

Trained non-human primate (NHP) studies have helped to establish foundational parameters and feasibility of discriminating between sensations arising from intracortical micro-stimulation (ICMS) with microelectrodes in the primary somatosensory cortex (PSC). These techniques have produced behavioral responses comparable to those produced by real tactile stimuli (Romo et al., 1998, 2000; O’Doherty et al., 2012; Klaes et al., 2014). Through alterations in amplitude and location of an ICMS, NHPs have demonstrated an ability to differentiate pressure, location, and timing on par with that of tactile stimulation. By varying frequency in ICMS $(10-30 \mathrm{~Hz}$, with a minimum absolute difference in comparative frequencies of $2 \mathrm{~Hz}$ ), NHPs showed similar accuracy to natural sensation produced by mechanical stimulation ( $80 \%$ vs. $89 \%$ accuracy) (Romo et al., 1998, 2000). O’Doherty et al. (2012) demonstrated that NHPs could differentiate between periodic and aperiodic pulse-trains in an active exploration task, suggesting that temporally patterned stimulation can lead to noticeable, unique sensations. Stimulation as low as $6 \mathrm{~Hz}$ produced perceptible sensations based on behavioral responses (Romo et al., 2000). They also identified that quickly adapting neurons were important in frequency discrimination, and discrimination between sensations was based on frequency alone. When slowly adapting neurons were stimulated, monkeys' performances decreased and were not comparable to mechanical stimulation (Romo et al., 2000).

While NHP studies have demonstrated that artificial sensation through cortical stimulation is achievable (O'Doherty et al., 2011; Tabot et al., 2013, 2015; Kim et al., 2015a,b; Overstreet et al., 2016), clinical studies in humans are needed to fully understand the subjective quality of sensations evoked by modulating these parameters and verify the generalizability. Direct electrical stimulation of PSC, with ICMS and surface electrodes, have yielded reliable and safe results (Flesher et al., 2016; Armenta Salas et al., 2018; Lee et al., 2018) and established basic stimulation parameters (Lee et al., 2018). Changes in amplitude and frequency are primarily perceived as increased intensity, with occasional enlargement of dermatomal areas involved, and rarely a change in perceptual quality (Armenta Salas et al., 2018; Lee et al., 2018). Amplitude increase was most consistent in producing increased intensity (Lee et al., 2018). A study utilizing ECoG electrodes at three frequencies (50, 75, and $100 \mathrm{~Hz}$ ) exemplified that sensation arising from stimulation at different frequencies can be differentiated in humans when there is at least a $25 \mathrm{~Hz}$ difference between frequencies and frequencies are greater than $50 \mathrm{~Hz}$ (Johnson et al., 2013). In addition, another high density ECoG study noted that perceiving somatosensation was inconsistent below $20 \mathrm{~Hz}$ for absolute perceptual threshold from a single stimuli (Lee et al., 2018). Although lower vibrotactile frequencies are better sensed in the skin (Vardar and Guclu, 2017), and have a wider range of responses (Griffin, 2012), earlier work by this group established a lower threshold in cortical stimulation of $20 \mathrm{~Hz}$ in which greater than $50 \%$ sensed the stimulation, and subject-described percepts reflected increased intensity and speed from increased cortical stimulation frequencies (Lee et al., 2018). Since the results of cortical stimulation on individual neurons is not well understood, the difference in frequency ranges and percepts likely reflects a difference in how cortical stimulation is interpreted compared to skin-sensed stimulation.

Going forward with BCI, establishing the psychometric thresholds for parameters like frequency will set the groundwork for what "degrees of freedom" are possible in subjects. Here we attempt to establish the perceptual limits of one specific parameter, frequency, during direct cortical stimulation of PSC using ECoG grids in humans with intact somatosensory pathways. We aim to estimate lower limits, and reliable degrees of freedom for frequency changes in stimulation parameters, for the purpose of using ECoG as a delivery method in somatosensory $\mathrm{BCI}$ in the future. We also aim to explore the usable limits for detecting the absolute difference between two frequencies.

\section{MATERIALS AND METHODS}

\section{Subjects and Implantation}

Three patients with intractable epilepsy, of normal intelligence on neuropsychiatric testing, normal somatosensation, and undergoing implantation of subdural ECoG with coverage over PSC, were enrolled in this study (see Table $\mathbf{1}$ for demographic details). These patients, as part of their care for epilepsy, required ECoG for seizure localization, and were to receive a craniotomy with access to the PSC hand region. S12 had a cavernous malformation in the parietal lobe. Based on the mapping of PSC, imaging, and results from invasive monitoring, the cavernoma was separate from the PSC hand area, and the seizure focus was not near PSC. S18 was found to have a seizure focus in the interhemispheric portion of the parietal lobe, also distinct from PSC. S30 was found to have seizure foci in the frontal and temporal lobes, also distinct from PSC. This study was approved by the USC Institutional Review Board and all subjects provided written consent. Surgical technique was standard, and has been described elsewhere (Lee et al., 2018), but briefly, a craniotomy was to be performed with access to the frontal, temporal, and parietal regions. Prior to surgery, the motor cortex hand area was identified based on anatomic landmarks, and the hand representation in PSC was marked, using neuronavigation software. During surgery, grids were placed to center over the PSC hand area. This area was not under direct visualization. Grids were high-density, "mini"ECoG grids (mECoG) with $2 \mathrm{~mm}$ contacts, with $1.2 \mathrm{~mm}$ exposed surface of platinum-iridium electrodes between silastic sheeting, spaced $3 \mathrm{~mm}$ apart from center-to-center (FG64CMP03, Ad-Tech Medical Instrument Corporation, Wisconsin, WI, United States), except in S12 where a standard spaced ECoG grid (sECoG) was used with $4 \mathrm{~mm}$ contacts, with $2.4 \mathrm{~mm}$ exposed surface, spaced $10 \mathrm{~mm}$ apart from center-to-center $(\mathrm{AU} 4 \times 5 \mathrm{P} 2$, Integra Life Sciences Corporation, New Jersey, United States). Following implantation, the grids were secured to the dura, 
TABLE 1 | Patient Demographics.

\begin{tabular}{|c|c|c|c|c|c|c|c|}
\hline Subject & Seizure foci & Radiographic abnormalities & $\begin{array}{c}\text { Epilepsy duration } \\
\text { (years) }\end{array}$ & $\begin{array}{c}\text { Age } \\
\text { (years) }\end{array}$ & $\operatorname{Sex}$ & $\begin{array}{l}\text { Dominant } \\
\text { hand }\end{array}$ & $\begin{array}{l}\text { Dermatome chosen } \\
\text { for stimulation }\end{array}$ \\
\hline S12 & Right parietal & $\begin{array}{l}\text { Prior surgery for right parietal } \\
\text { cavernous malformation }\end{array}$ & 3 & 25 & $\mathrm{M}$ & $\mathrm{R}$ & Digit 5 medial surface \\
\hline S18 & $\begin{array}{l}\text { Posterior interhemispheric strip, } \\
\text { lateral parietal }\end{array}$ & N/A & 11 & 32 & $\mathrm{~F}$ & $\mathrm{R}$ & Medial palm \\
\hline S30 & $\begin{array}{l}\text { Left interhemispheric frontal } \\
\text { cortex in area of } \\
\text { encephalomalacia }\end{array}$ & $\begin{array}{l}\text { Left frontal/temporal } \\
\text { encephalomalacia }\end{array}$ & 11 & 24 & $\mathrm{~F}$ & $\mathrm{R}$ & Medial palm and wrist \\
\hline
\end{tabular}

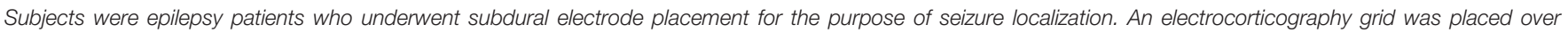

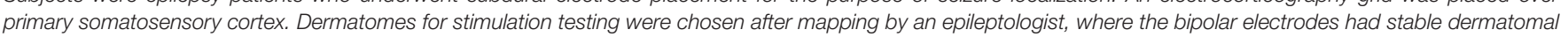
percepts upon multiple repeat stimulations.

tunneled through the scalp, and secured to the scalp with suture. The dura was closed, bone replaced, and scalp closed; the patient was transported to the epilepsy monitoring unit in the intensive care unit (ICU).

\section{Experimental Set Up}

Location of the implanted grid was confirmed by imaging with a computed tomography scan fused to a preoperative magnetic resonance imaging scan (Figure 1). Superimposition of grid placement was made using Freesurfer and Statistical Parametric Mapping software SPM12 with the img_pipe package described in Hamilton et al. (2017). Functional location of electrodes was confirmed by mapping with cortical stimulation while subjects were in the ICU. Electrode pairs were stimulated with a range of amplitudes, at the discretion of the epileptologist, between 0.5 and $12 \mathrm{~mA}$, with a frequency of $50 \mathrm{~Hz}$, pulse-width of $250 \mu \mathrm{s}$, and duration of $1 \mathrm{~s}$. Areas with pure sensory responses (self-reported by the subject), underwent steady increases in amplitude until motor responses were noted. Following mapping, electrode pairs with sensory only responses at $4 \mathrm{~mA}$ were retested 25 times to confirm that repeat stimulation did not (1) alter the percept by the subject, (2) alter the location or region of perception, (3) result in motor activity, (4) cause seizures or seizure-like activity, or (5) cause discomfort. All subjects had at least one electrode pair that met these criteria. If more than one electrode pair met these criteria, there was a preference for the ventral side of the hand and for digits on the lateral side of the hand over the medial (see Table 1). This electrode pair was then chosen for repeat stimulation with our paradigm.

Subjects explored a 2-dimensional space consisting of a sheet with two circles on it, which the subject placed at a comfortable distance for exploring with the hand contralateral to the implanted grid. The two circles corresponded to two different stimulation frequencies. As the subject moved one hand over the two circles, an epileptologist stimulated PSC with the associated frequency using an FDA-approved, clinically available stimulator (Natus Neurology Incorporated, Warwick, United States). The current, pulse-width, and pulse-duration were held stable $(4 \mathrm{~mA}$, $250 \mu \mathrm{s}$, and $1 \mathrm{~s}$, respectively). In a two-alternative forcedchoice task design, subjects were instructed to report which circle corresponded to the higher frequency, whether they were "guessing" (meaning they were not sure which one was the higher frequency), and whether they felt both stimulations (see Figure 2 for experimental setup). Frequencies included 20, 30, 40, 50, 60, and $100 \mathrm{~Hz}$ and were chosen pseudorandomly. The stimulator did not have parameters between 60 and $100 \mathrm{~Hz}$. Because subjects moved at their own speed, the time between stimuli ranged from 1 to $6 \mathrm{~s}$. Statistical analysis was performed using Matlab software (The Mathworks, Natick, MA, United States). Thresholds and differences in detection were compared using Fisher's exact test.

\section{RESULTS}

Electrodes used for stimulation were determined after cortical mapping and somatosensory percepts were reported by the subject and remained stable after repeat stimulations ( $>50$ stimulations). Stimulation parameters included pulse-width of $250 \mu \mathrm{s}$, duration of $1 \mathrm{~s}$, and a square-wave, and were chosen based on prior literature (Lee et al., 2018) and to minimize the interplay of the other parameters with frequency (i.e., low, but reliably detected on 25 repeat stimulations at $50 \mathrm{~Hz}$ ). Amplitude was chosen to be the lowest value that elicited reliable somatosensory percepts on the 50 prior stimulations with a $50 \mathrm{~Hz}$ frequency, and that did not elicit motor activity (2 $\mathrm{mA}$ for S12, $3 \mathrm{~mA}$ for $\mathrm{S} 18$, and $5 \mathrm{~mA}$ for S30). Selected dermatomes were the medial surface of digit 5 for S12, medial surface of the palm for S18, and medial palm and wrist for S30 (Table 1). Twenty-five trials were completed for S12 and 50 trials for S18 and S30. For S12, 50 trials were planned, however, the patient chose to stop half-way through testing due to fatigue. No adverse events occurred. With increased frequency, patients described the sensation as "more intense," "faster," and "faster buzzing."

\section{Overall Accuracy}

Altogether, participants identified the higher frequency with $89.76 \%$ accuracy. To explore whether the first stimulation might alter the perception of the second stimulation, trials were grouped based on which condition occurred first. Correct responses were statistically equivalent between these groups $(91.9 \%$ when the higher frequency occurred first vs. $86.8 \%$ when it occurred second; $p=0.39$, Fisher's exact test). The accuracy of trials broken down into the individual categories is included in Figure 3. 


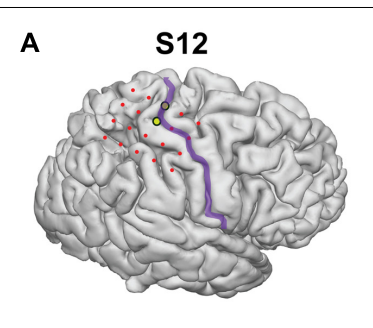

$\mathbf{B}$

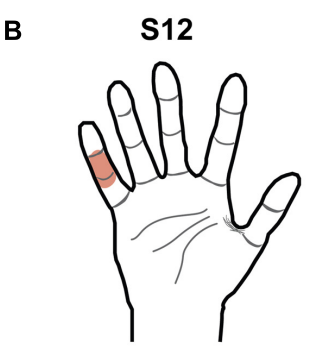

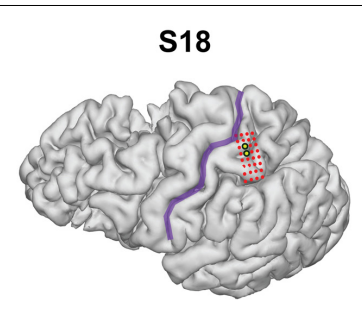

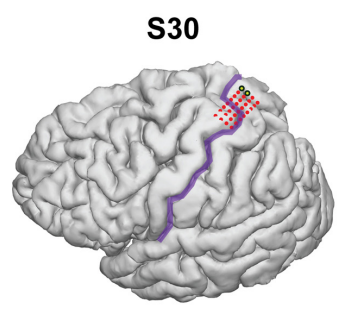

S18

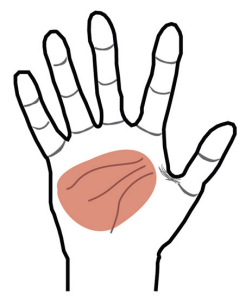

$\mathbf{S} 30$

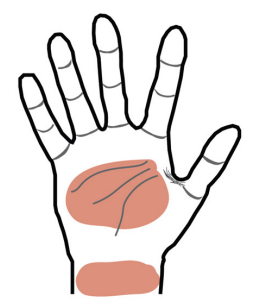

FIGURE 1 | Electrocorticography grid placement. (A) 3-dimensional images of each subject's brain from a magnetic resonance image, with the location of the electrodes superimposed. The central sulcus is outlined in purple and electrodes of the grid are shown in red. The electrodes chosen for stimulation in this experimental model are highlighted in yellow. S12 had electrodes with $1 \mathrm{~cm}$ of spacing, and S18 and S30 had electrodes with $3 \mathrm{~mm}$ of spacing (center-to-center). (B) The dermatomal distribution of the percepts used for testing after grid mapping for each subject.

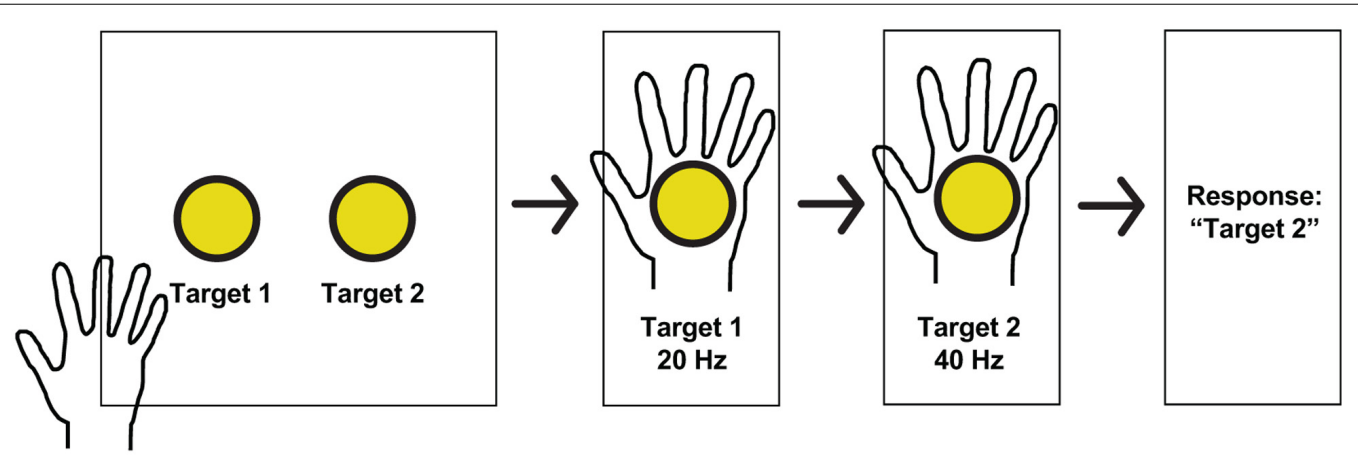

FIGURE 2 | Experimental paradigm and hand receptive fields. Schematic of a typical session. Subjects received stimulation at one of two pseudorandomly determined frequencies when searching for targets in a 2-dimensional space, and reported which target had the higher frequency, whether or not they guessed, and whether or not both stimulations were perceived.

Next, we focused on the absolute difference between two frequencies (see Figures 4, 5). When the frequency difference was equal to $10 \mathrm{~Hz}$, the mean accuracy was $74.29 \%$ (S12 71.4\%; S18 88.9\%; S30 68.4\%, see Figure 5) compared to a difference larger than $10 \mathrm{~Hz}$, where accuracy increased to $95.65 \%(p<0.001$, individually: S12 100\%; S18 100\%; S30 87.1\%). For S12 and S18, incorrect responses only occurred at a difference of $10 \mathrm{~Hz}$, when both frequencies were equal to or less than $40 \mathrm{~Hz}$.

Using $40 \mathrm{~Hz}$ as a cutoff of lower frequencies and higher frequencies, we examined whether small absolute differences were easier to differentiate at lower frequencies or higher frequencies. Separating the trials into those with both frequencies $40 \mathrm{~Hz}$ or less vs. those with either frequency greater than $40 \mathrm{~Hz}$, accuracy was $77.8 \%$ vs. $93.0 \%(p<0.05)$. However, this difference was largely explained by the larger absolute differences of frequencies above $40 \mathrm{~Hz}$. Comparing the absolute difference of $20 \mathrm{~Hz}$ or less (since the frequencies at or below $40 \mathrm{~Hz}$ had a max absolute difference of $20 \mathrm{~Hz}$ ) the accuracy was $77.8 \%$ vs. $80.0 \%$ ( $p=1.0$ ) for below $40 \mathrm{~Hz}$ and above $40 \mathrm{~Hz}$, respectively. When isolating the trials in which the frequency difference was $10 \mathrm{~Hz}$, and then separating them into those with both frequencies $40 \mathrm{~Hz}$ or less vs. those with one or both greater than $40 \mathrm{~Hz}$, accuracy was $77.3 \%$ vs. $69.2 \%(p=0.69)$, respectively. At a frequency difference of $20 \mathrm{~Hz}$, accuracy was $80 \%$ when both frequencies were $40 \mathrm{~Hz}$ or less compared to $88.2 \%$ when at least one was greater than $40 \mathrm{~Hz}(p=0.66)$ (Figure 5). Frequency differences of $20 \mathrm{~Hz}$ had an accuracy of $86.4 \%$, and differences of $30 \mathrm{~Hz}$ had an accuracy of $95.0 \%$. All other differences had an accuracy of 100\% (see Figure 5).

\section{Patient Reported "Guessing"}

Subjects reported when they were "guessing," defined as when they could not tell which stimulus was higher. Of all trials, guessing occurred in $18.1 \%$ of trials. When subjects reported 


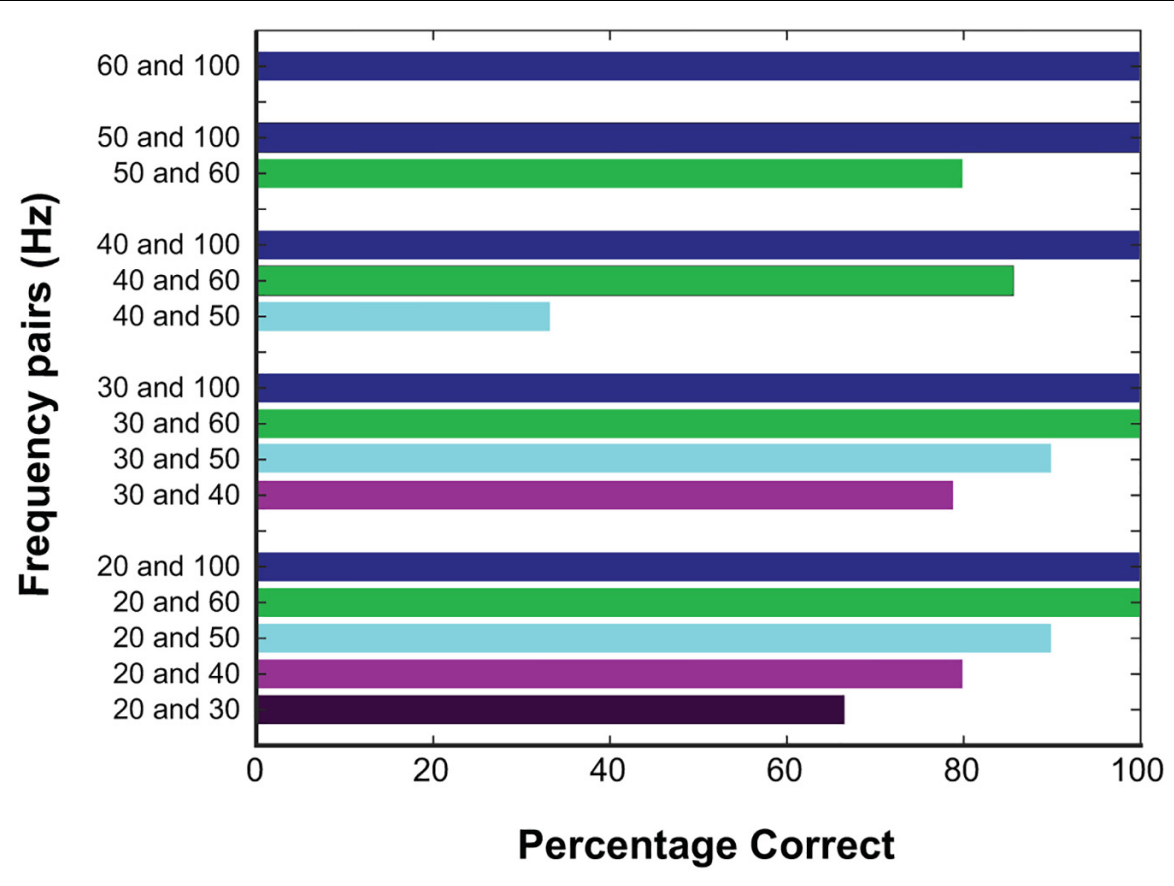

FIGURE 3 | Responses at different frequencies tested. Percentage correct for all trials based on the frequencies being compared, color coded based on the higher frequency. Correct responses were high with larger frequency differences.

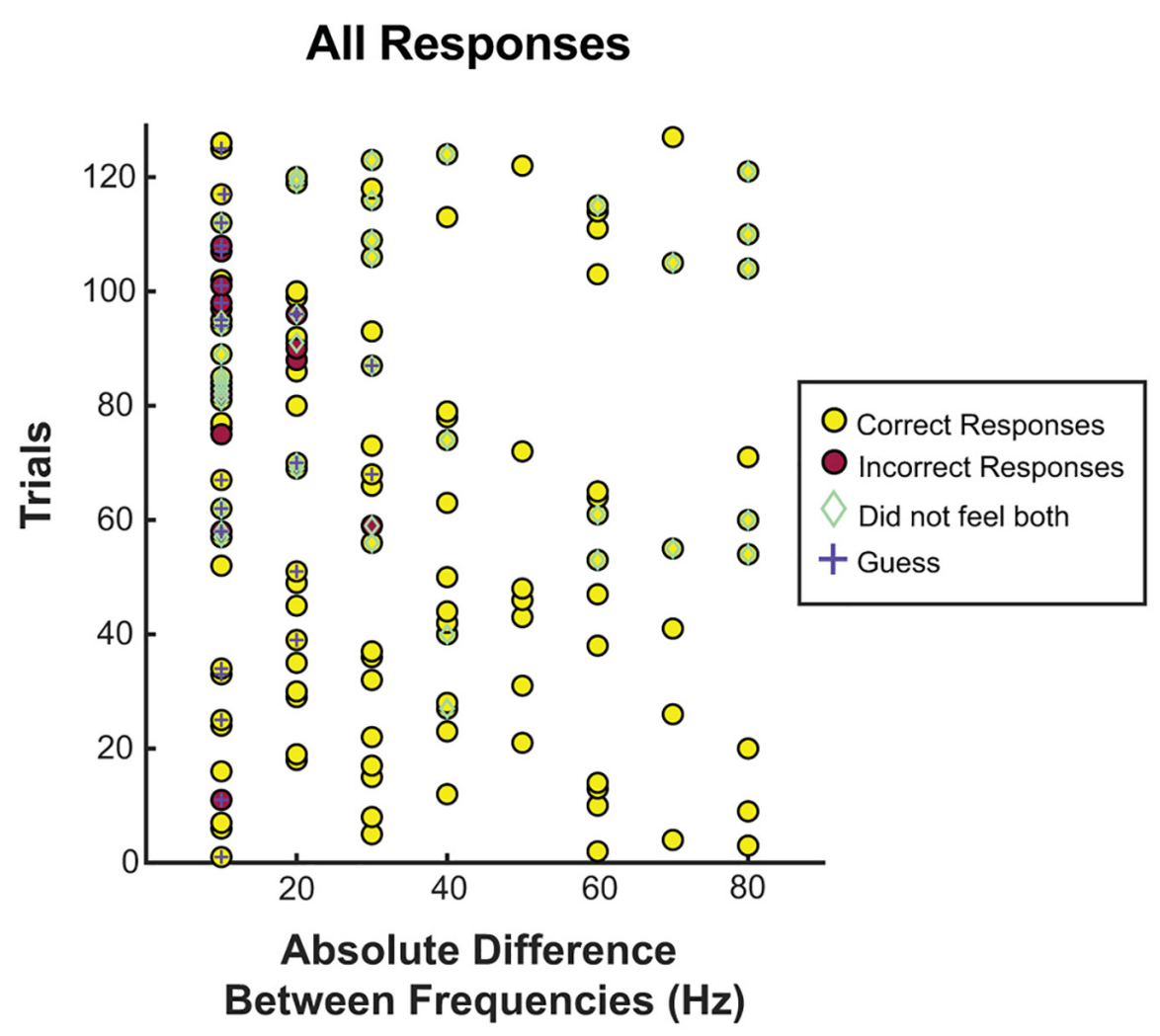

FIGURE 4 | Correct responses organized by absolute difference between two frequencies. All trials separated by the absolute difference in frequency. Correct and incorrect responses, guessing, and whether or not the patient felt both stimuli were recorded for each frequency difference. Most incorrect trials, guessing, and inability to feel both frequencies occurred at or below a difference of $20 \mathrm{~Hz}$. 


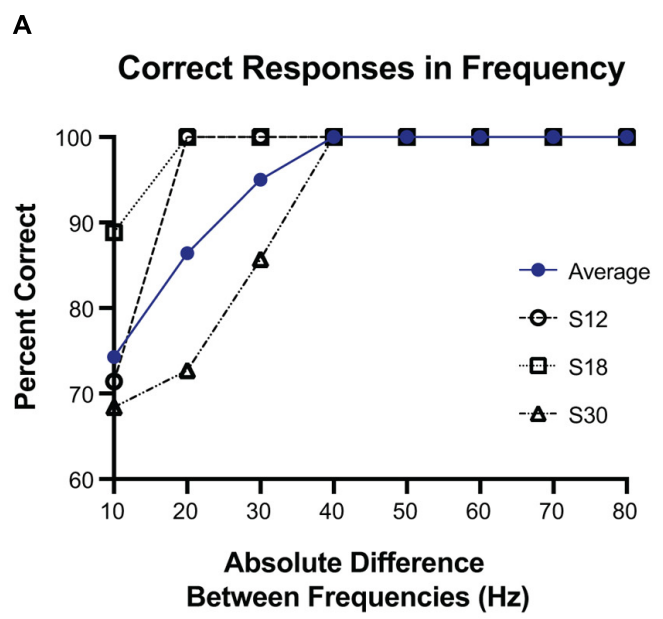

B

\section{Accuracy Above and Below $40 \mathrm{~Hz}$}

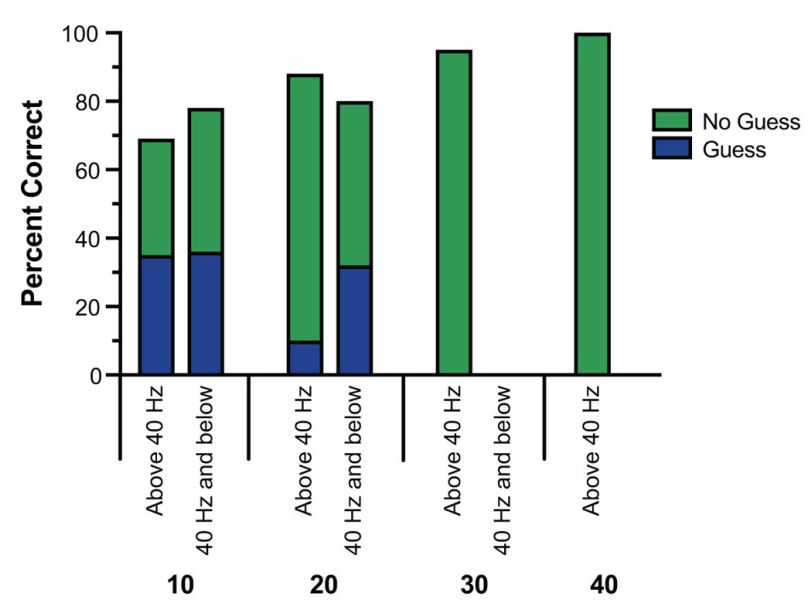

Absolute Difference

Between Frequencies ( $\mathrm{Hz})$

FIGURE 5 | Accuracy at tested frequency differences. (A) Overall accuracy for each subject, at each frequency difference. The average of their results is in blue. Above a $30 \mathrm{~Hz}$ difference, accuracy was 100\%, and well above chance at or below $30 \mathrm{~Hz}$. (B) Response accuracy and guessing rate when the tested frequencies were both $40 \mathrm{~Hz}$ and below vs. when one or more of the tested frequencies was above $40 \mathrm{~Hz}$. Here the blue and green represent which portions of correct responses were self-reported as a guess, or not a guess, respectively. Overall, subjects were equally accurate regardless of whether the frequencies were above or below $40 \mathrm{~Hz}$, and were more likely to guess if the difference was $10 \mathrm{~Hz}$.

guessing, accuracy was $69.6 \%$ vs. $94.2 \%$ when they did not report guessing $(p<0.01)$. Limited to trials in which the difference was $20 \mathrm{~Hz}$ or less, guessing occurred $36.8 \%$ of the time, vs. $2.9 \%$ of the time when the difference was greater than $20 \mathrm{~Hz}(p<0.001)$. Accuracy for only trials in which the difference was $20 \mathrm{~Hz}$ or less was $66.7 \%$ when guessing vs. $86.1 \%$ when not guessing $(p=0.10)$.

Further, to examine if guessing was more frequent when the frequencies were both low vs. when they were high, we split the $20 \mathrm{~Hz}$ differences into those both at or below $40 \mathrm{~Hz}$ vs. the rest (see Figure 4). For a difference of $20 \mathrm{~Hz}$ or less, when both frequencies were at or below $40 \mathrm{~Hz}$, guessing occurred $48.1 \%$ of the time vs. $26.7 \%$ when one or more frequencies was above $40 \mathrm{~Hz}$ $(p=0.10)$, and correct responses were given $77.8 \%$ of the time at or below $40 \mathrm{~Hz}$ vs. $80.0 \%$ of the time above $40 \mathrm{~Hz}(p=1.0)$.

When the difference was equal to $10 \mathrm{~Hz}$, guessing occurred $48.6 \%$ of the time, vs. $6.5 \%$ of the time when the frequency difference was greater than $10 \mathrm{~Hz}(p<0.001)$. Among trials with a difference of $10 \mathrm{~Hz}$, accuracy was $64.7 \%$ when guessing vs. $83.3 \%$ when not guessing $(p=0.26)$. Again, we examined if having both frequencies lower than $40 \mathrm{~Hz}$ affected the rate of accuracy or guessing among those that were $10 \mathrm{~Hz}$ apart. No difference was seen among subjects' rate of guessing when both frequencies were at or below $40 \mathrm{~Hz}$ vs. one or more above $40 \mathrm{~Hz}$ ( $46.1 \%$ vs. $50.0 \%, p=1.0$ ), or subjects' accuracy when guessing $(77.2 \%$ vs. $69.2 \%, p=0.70)$.

\section{Thresholds of Detection}

To understand the lower limits of detection thresholds, subjects were asked to report on whether they felt both stimulations, of which $69.3 \%$ of trials were reported as perceived for both stimulations (see Figure 4). Of the $30.7 \%$ in which one or more stimulations were not perceived, $82.1 \%$ had one or both frequencies at or below $30 \mathrm{~Hz}$, and $92.3 \%$ had one or both frequencies at or below $40 \mathrm{~Hz}$. Correct responses were not significantly different when subjects felt both stimulations $(89.8 \%$ when both were felt vs. $89.7 \%$ when one or both were not felt). No difference was found in whether both stimulations were felt when split for both stimulation frequencies being below $50 \mathrm{~Hz}$ vs. both being above $50 \mathrm{~Hz}(60.0 \%$ vs. $75.3 \%$, $p=0.079$ ) or both being below $40 \mathrm{~Hz}$ vs. both being above $40 \mathrm{~Hz}$ (63.0\% vs. $71.0 \%, p=0.483)$.

\section{DISCUSSION}

Here we build upon prior work using ECoG electrodes to establish the thresholds of functionality for somatosensory BCI by adjusting frequency and discerning discrimination thresholds. The ability to detect a difference in frequency from cortical stimulation using an ECoG grid adds an adjustable parameter to the prospect of somatosensory BCI. To this end, we sought to explore the basic psychometric parameters of frequency discrimination in epilepsy patients with already implanted ECoG grids over PSC. Subjectively, when frequency increased, patients perceived a more intense stimulus, similar to other stimulation studies in humans (Johnson et al., 2013; Hiremath et al., 2017; Lee et al., 2018). Even when subjects reported guessing on a forced choice between stimulations of two different frequencies, accuracy was $69.6 \%$. Finally, we show detection of absolute differences between stimulation frequencies above chance at 
$10 \mathrm{~Hz}$, with reliable differentiation of frequencies at or above $20 \mathrm{~Hz}$ and near perfect detection at or above $40 \mathrm{~Hz}$.

Prior ECoG work with cortical stimulation tested frequencies of 50,75 , and $100 \mathrm{~Hz}$ and noted that patients were able to identify and differentiate between sensations elicited by these frequencies (Johnson et al., 2013). Our own work showed that intensity was altered with an increase in frequency, but detecting differences was not explicitly tested (Lee et al., 2018). These studies investigating the frequency component of stimulation have mostly studied the feasibility and subjective quality of modulating frequency to produce different sensations, as well as the threshold required for somatosensory percepts (Johnson et al., 2013; Hiremath et al., 2017; Lee et al., 2018). Prior studies in microelectrode stimulation have focused on amplitude, demonstrating alterations in somatosensory category, with the higher amplitudes leading to more proprioceptive sensations, and lower amplitudes leading to cutaneous sensations (Armenta Salas et al., 2018). Further intracortical stimulation with microelectrodes in humans suggested that the "just-noticeable difference" (JND) of amplitude is around $15.4 \pm 3.9 \mu \mathrm{A}$ with a detection threshold of $39.4 \mu \mathrm{A}$ (Flesher et al., 2016).

To establish similar baseline parameters in frequency alterations, we built off prior work. With preliminary testing we noted that frequencies below $20 \mathrm{~Hz}$ were generally undetectable (Lee et al., 2018), and thus explored $10 \mathrm{~Hz}$ differences from 20 to $100 \mathrm{~Hz}$ (except 70-90 Hz due to technical constraints). Above a $30 \mathrm{~Hz}$ difference, accuracy was $100 \%$, with a high degree of accuracy at all tested differences below $30 \mathrm{~Hz}$. Frequency differences of $20 \mathrm{~Hz}$ led to an accuracy of $86.4 \%$, and differences of $10 \mathrm{~Hz}$ were still well above chance at $74.3 \%$. S12 and S18 had only two incorrect responses each, both at a $10 \mathrm{~Hz}$ difference, with both frequencies at or below $40 \mathrm{~Hz}$. S30 was more varied, but had most incorrect responses at a difference of $10 \mathrm{~Hz}(60 \%)$, and all were when both frequencies were less than $60 \mathrm{~Hz}$.

Although it was more difficult to detect a difference of $10 \mathrm{~Hz}$ compared to 20 or $30 \mathrm{~Hz}$, accuracy at a $10 \mathrm{~Hz}$ difference was well above chance, and thus we estimate a JND below $10 \mathrm{~Hz}$ for cortical stimulation through ECoG electrodes. One exception to this, in Figure 3, $40 \mathrm{~Hz}$ vs. $50 \mathrm{~Hz}$ has a $33.3 \%$ accuracy, and may reflect a limited number of trials at that frequency pair, or reflect a true difference. The edges of detection for Meissener corpuscles (flutter sensations at frequencies 10-60 Hz) and Pacinian afferents (vibrational sensations 60-400 Hz) are right around 40 and $50 \mathrm{~Hz}$ (Mountcastle et al., 1969), and therefore this may reflect a difficulty in this frequency range for both cortical and peripheral stimulation. Further testing may be warranted.

Weber's law would indicate that the JND would increase proportionally to the increase in the base frequency. Indeed, Weber's law is noted in studies comparing tactile somatosensation (Francisco et al., 2017), however it is not clear if this follows in cortical stimulation. Limited by the testing capabilities of our stimulator, we were unable to explore smaller differences, and thus unable to find a true JND, or the adherence of PSC stimulation to Weber's law. In a NHP microstimulation study, the JND was $3.73 \mathrm{~Hz}$ with cortical stimulation (Romo et al., 2000), which may be closer to the real JND or may represent a difference in species, electrode type, or both. Compared to vibrational frequencies of mechanical stimuli, the JND was similar between the two $(2.88 \mathrm{~Hz}$ for mechanical stimuli). JND could be different with ECoG electrode stimulation vs. microelectrode stimulation given changes in the spread of electricity (bipolar vs. unipolar), size of the electrode (mm vs. $\mu \mathrm{m}$ ), or other differences (e.g., mA vs. $\mu \mathrm{A}$ ). The JND estimated in this study $(<10 \mathrm{~Hz})$ is not far from the JNDs reported for microelectrode stimulation and mechanical stimuli, and although it may abide by Weber's law, all that we can conclude here is that between 50 and $60 \mathrm{~Hz}$ (the largest base frequency with the smallest absolute difference), accuracy was well above chance at $80 \%$. Since microelectrode, macroelectrode, and mechanical stimulation appear to have similar JNDs, it may be that the encoding of vibrational frequencies is tied to the frequency of the electrical activity in PSC whether from peripheral nerves or direct stimulation. Indeed, lower vibrational frequencies of mechanical stimuli $(<100 \mathrm{~Hz})$ have been seen to stimulate PSC neuron spike rates (Mountcastle et al., 1969; Salinas et al., 2000; Hernandez et al., 2005; Luna et al., 2005). Further testing with stimulators with more finely tuned adjustments will be necessary in the future, and may need to rely on concepts that compare tactile and cortical stimulation thresholds like "psychometric equivalence functions," already shown to be able to estimate these thresholds in amplitude and frequency stimulations in rodents (Devecioglu and Guclu, 2005).

Subject-reported guessing was also evaluated, showing a low rate of guessing across all trials at $18.1 \%$. Most guessing occurred at the lower range of differences, 10 and $20 \mathrm{~Hz}$, however, accuracy was not significantly altered, and correct responses were above $50 \%$. This result indicates that despite self-reports, subjects were still able to differentiate between frequencies. Similarly, subjects reported they could not feel both stimulations approximately $1 / 3$ of the time. Again, this outcome did not diminish correct responses, which were similar when comparing those in which they reported feeling both to those in which they did not (see Figure 3). Ninety-two percent of the time that subjects did not sense the stimulation, one frequency was at or below $40 \mathrm{~Hz}$. Taken together, accuracy decreased and guessing increased when both frequencies were less than $30 \mathrm{~Hz}$, and inconsistencies were seen at 30 and $40 \mathrm{~Hz}$. Given these results, $40 \mathrm{~Hz}$ is likely the threshold for consistent sensation, and would be a reasonable lower limit for a somatosensory BCI system. From these results, an ECoG BCI system would likely be able to manipulate frequencies at $20 \mathrm{~Hz}$ differences from $40 \mathrm{~Hz}$ and above, with a high degree of reliability. With training and more fine adjustments, differences of $10 \mathrm{~Hz}$ would likely be sufficient. We did see a difference between subjects, with one subject showing decreased accuracy above $30 \mathrm{~Hz}$, suggesting intersubject variability. Additionally, the thresholds were based on fixed parameters of amplitude, pulsewidth, and pulse-duration, all factors which independently (Lee et al., 2018), and jointly influence thresholds (Devecioglu and Guclu, 2005). Likely an increased amplitude or pulse-duration would alter the thresholds of frequency discrimination, as well as the perception of the stimulus [as evidenced by changes in the strength of the stimulus noted by Lee et al. (2018)]. A great deal more testing in future studies will be required to elucidate the psychophysical results of the combining parameter variations 
and a future BCI system will likely need a range of possible frequencies to adjust for individual variation.

This study has several limitations. First, the stimulator used, an FDA-approved clinical stimulator, can only test differences as small as $10 \mathrm{~Hz}$. Therefore, while the "just-noticeable" difference most likely lies below $10 \mathrm{~Hz}$, we were unable to stimulate smaller changes given that the stimulator settings are unalterable (see Figure 4). Similarly, the parameters are limited, without options between 60 and $100 \mathrm{~Hz}$. Testing was carried out in the ICU, limiting time and control, and preventing more trials and further paradigms. Subjects all suffered from epilepsy, which may alter cortical networks in PSC. However, our subjects were still able to perceive sensation in a consistent dermatome upon stimulation and had no known pathology affecting PSC. The other stimulation parameters were not consistent between patients (but rather consistent within patients), introducing bias. Electrode size was different for S12 than S18 and S30, which may have introduced differences into the spread of electricity. Each session only took place in a single day and the results are therefore not generalizable to chronic stimulation. The stability of chronic stimulation for producing somatosensation is unknown, however, reports of chronic stimulation of motor cortex in NHPs shows stability of mapping (Craggs et al., 1976), the safety profile from chronically implanted, stimulating, and surface electrodes in responsive neural stimulators is quite robust (Heck et al., 1976; Bergey et al., 2015; Lee et al., 2015). ICMS use in PSC exhibits stability at 10-12 weeks, without any safety concerns. Since ICMS is invasive, it is likely that the safety profile would be similar or better, but the decay may be more severe (Callier et al., 2015; Chen et al., 2015).

Overall, this study uses ECoG grids to investigate the frequency component of cortical stimulation for use in BCI systems. We estimate a JND value near or below $10 \mathrm{~Hz}$, and show that even when guessing, subjects could correctly identify the higher frequency better than chance. Furthermore, these findings suggest reliable discrimination above $40 \mathrm{~Hz}$, with a difference between frequencies of $20 \mathrm{~Hz}$ or above. Future BCI systems

\section{REFERENCES}

Aflalo, T., Kellis, S., Klaes, C., Lee, B., Shi, Y., Pejsa, K., et al. (2015). Neurophysiology. Decoding motor imagery from the posterior parietal cortex of a tetraplegic human. Science 348, 906-910. doi: 10.1126/science.aaa5417

Andersen, R. A., Hwang, E. J., and Mulliken, G. H. (2010). Cognitive neural prosthetics. Annu. Rev. Psychol. 61, C1-C3. doi: 10.1146/annurev.psych.093008. 100503

Andersen, R. A., Musallam, S., and Pesaran, B. (2004). Selecting the signals for a brain-machine interface. Curr. Opin. Neurobiol. 14, 720-726. doi: 10.1016/j. conb.2004.10.005

Armenta Salas, M., Bashford, L., Kellis, S., Jafari, M., Jo, H., Kramer, D., et al. (2018). Proprioceptive and cutaneous sensations in humans elicited by intracortical microstimulation. eLife 7:e32904. doi: 10.7554/eLife.32904

Baumgartner, C., Barth, D. S., Levesque, M. F., and Sutherling, W. W. (1991). Functional anatomy of human hand sensorimotor cortex from spatiotemporal analysis of electrocorticography. Electroencephalogr. Clin. Neurophysiol. 78, 56-65. doi: 10.1016/0013-4694(91)90019-Z

Bergey, G. K., Morrell, M. J., Mizrahi, E. M., Goldman, A., King-Stephens, D., Nair, D., et al. (2015). Long-term treatment with responsive brain stimulation utilizing cortical stimulation to produce artificial sensation can utilize frequency to produce a wider range of percepts, empowering users to make better use of artificial sensations.

\section{ETHICS STATEMENT}

This study was carried out in accordance with the recommendations of the University of Southern California Health Sciences Campus Institutional Review Board with written informed consent from all subjects. All subjects gave written informed consent in accordance with the Declaration of Helsinki. The protocol was approved by the University of Southern California Health Sciences Campus Institutional Review Board.

\section{AUTHOR CONTRIBUTIONS}

DK, BL, SK, and CL conceived the original ideas and experiments. $\mathrm{BL}$ and DK carried out the experiments. All authors interpreted the data, edited the manuscript, provided critical feedback, and helped to shape the research and analysis. DK, KL-F, CL, BK, and SK led the writing of the manuscript.

\section{FUNDING}

We wish to acknowledge the generous support of Cal-BRAIN: A Neurotechnology Program for California, National Center for Advancing Translational Science (NCATS) of the U.S. National Institutes of Health (KL2TR001854), National Institutes of Health (R25 NS099008-01), the Neurosurgery Research and Education Foundation (NREF), the Tianqiao and Chrissy Chen Brain-Machine Interface Center at Caltech, the Boswell Foundation and the Della Martin Foundation, and the University of Southern California Neurorestoration Center. None of the listed sources of funding had a role in study design, collection, analysis, and interpretation of data, or writing of the manuscript.

in adults with refractory partial seizures. Neurology 84, 810-817. doi: 10.1212/ wnl.0000000000001280

Bundy, D. T., Pahwa, M., Szrama, N., and Leuthardt, E. C. (2016). Decoding three-dimensional reaching movements using electrocorticographic signals in humans. J. Neural. Eng. 13:026021. doi: 10.1088/1741-2560/13/2/02 6021

Callier, T., Schluter, E. W., Tabot, G. A., Miller, L. E., Tenore, F. V., and Bensmaia, S. J. (2015). Long-term stability of sensitivity to intracortical microstimulation of somatosensory cortex. J. Neural. Eng. 12:056010. doi: 10.1088/1741-2560/12/ $5 / 056010$

Chen, K. H., Dammann, J. F., Boback, J. L., Tenore, F. V., Otto, K. J., Gaunt, R. A., et al. (2015). The effect of chronic intracortical microstimulation on the electrode-tissue interface. J. Neural. Eng. 11:026004. doi: 10.1088/1741-2560/ $11 / 2 / 026004$

Chestek, C. A., Gilja, V., Blabe, C. H., Foster, B. L., Shenoy, K. V., Parvizi, J., et al. (2013). Hand posture classification using electrocorticography signals in the gamma band over human sensorimotor brain areas. J. Neural. Eng. 10:026002. doi: 10.1088/1741-2560/10/2/026002

Collins, K. L., Guterstam, A., Cronin, J., Olson, J. D., Ehrsson, H. H., and Ojemann, J. G. (2017). Ownership of an artificial limb induced by electrical 
brain stimulation. Proc. Natl. Acad. Sci. U.S.A. 114, 166-171. doi: 10.1073/pnas. 1616305114

Craggs, M. D., Rushton, D. N., and Clayton, D. G. (1976). The stability of the electrical stimulation map of the motor cortex of the anesthetized baboon. Brain 99, 575-600. doi: 10.1093/brain/99.3.575

Devecioglu, I., and Guclu, B. (2005). Psychophysical correspondence between vibrotactile intensity and intracortical microstimulation for tactile neuroprostheses in rats. J. Neural. Eng. 14:016010. doi: 10.1088/1741-2552/14/1/016010

Fifer, M. S., Acharya, S., Benz, H. L., Mollazadeh, M., Crone, N. E., and Thakor, N. V. (2012). Toward electrocorticographic control of a dexterous upper limb prosthesis: building brain-machine interfaces. IEEE Pulse 3, 38-42. doi: 10. 1109/MPUL.2011.2175636

Flesher, S. N., Collinger, J. L., Foldes, S. T., Weiss, J. M., Downey, J. E., Tyler-Kabara, E. C., et al. (2016). Intracortical microstimulation of human somatosensory cortex. Sci. Transl. Med. 8:361ra141. doi: 10.1126/scitranslmed.aaf 8083

Francisco, E., Tannan, V., Zhang, Z., Holden, J., and Tommerdahl, M. (2017). Vibrotactile amplitude discrimination capacity parallels magnitude changes in somatosensory cortex and follows Weber's Law. Exp. Brain Res. 191, 49-56. doi: 10.1007/s00221-008-1494-6

Griffin, M. J. (2012). Frequency-dependence of psychophysical and physiological responses to hand-transmitted vibration. Ind. Health 50, 354-369. doi: 10.2486/ indhealth.ms 1379

Hamilton, L. S., Chang, D. L., Lee, M. B., and Chang, E. F. (2017). Semi-automated anatomical labeling and inter-subject warping of high-density intracranial recording electrodes in electrocorticography. Front. Neuroinform. 11:62. doi: 10.3389/fninf.2017.00062

Heck, C. N., King-Stephens, D., Massey, A. D., Nair, D. R., Jobst, B. C., Barkley, G. L., et al. (1976). Two-year seizure reduction in adults with medically intractable partial onset epilepsy treated with responsive neurostimulation: final results of the RNS System Pivotal trial. Epilepsia 55, 432-441. doi: 10.1111/epi. 12534

Hernandez, A., Zainos, A., and Romo, R. (2005). Neuronal correlates of sensory discrimination in the somatosensory cortex. Proc. Natl. Acad. Sci. U.S.A. 97, 6191-6196. doi: 10.1073/pnas.120018597

Hiremath, S. V., Tyler-Kabara, E. C., Wheeler, J. J., Moran, D. W., Gaunt, R. A., Collinger, J. L., et al. (2017). Human perception of electrical stimulation on the surface of somatosensory cortex. PLoS One 12:e0176020. doi: 10.1371/journal. pone. 0176020

Hollins, M., and Risner, S. R. (2016). Evidence for the duplex theory of tactile texture perception. Percept. Psychophys. 62, 695-705. doi: 10.3758/bf03206916

Johnson, L. A., Wander, J. D., Sarma, D., Su, D. K., Fetz, E. E., and Ojemann, J. G. (2013). Direct electrical stimulation of the somatosensory cortex in humans using electrocorticography electrodes: a qualitative and quantitative report. J. Neural. Eng. 10:036021. doi: 10.1088/1741-2560/10/3/036021

Kim, S., Callier, T., Tabot, G. A., Gaunt, R. A., Tenore, F. V., and Bensmaia, S. J. (2015a). Behavioral assessment of sensitivity to intracortical microstimulation of primate somatosensory cortex. Proc. Natl. Acad. Sci. U.S.A. 112, 1520215207. doi: 10.1073/pnas.1509265112

Kim, S., Callier, T., Tabot, G. A., Tenore, F. V., and Bensmaia, S. J. (2015b). Sensitivity to microstimulation of somatosensory cortex distributed over multiple electrodes. Front. Syst. Neurosci. 9:47. doi: 10.3389/fnsys.2015.00047

Klaes, C., Shi, Y., Kellis, S., Minxha, J., Revechkis, B., and Andersen, R. A. (2014). A cognitive neuroprosthetic that uses cortical stimulation for somatosensory feedback. J. Neural. Eng. 11:056024. doi: 10.1088/1741-2560/11/5/056024

Lee, B., Kramer, D., Armenta Salas, M., Kellis, S., Brown, D., Dobreva, T., et al. (2018). Engineering artificial somatosensation through cortical stimulation in humans. Front. Syst. Neurosci. 12:24. doi: 10.3389/fnsys.2018.00024

Lee, B., Liu, C. Y., and Apuzzo, M. L. (2013). A primer on brain-machine interfaces, concepts, and technology: a key element in the future of functional neurorestoration. World Neurosurg. 79, 457-471. doi: 10.1016/j.wneu.2013.01. 078

Lee, B., Zubair, M. N., Marquez, Y. D., Lee, D. M., Kalayjian, L. A., Heck, C. N., et al. (2015). A Single-center experience with the NeuroPace RNS system: a review of techniques and potential problems. World Neurosurg. 84, 719-726. doi: 10.1016/j.wneu.2015.04.050

Luna, R., Hernandez, A., Brody, C. D., and Romo, R. (2005). Neural codes for perceptual discrimination in primary somatosensory cortex. Nat. Neurosci. 8 , 1210-1219. doi: $10.1038 / \mathrm{nn} 1513$

Mountcastle, V. B., Talbot, W. H., Sakata, H., and Hyvarinen, J. (1969). Cortical neuronal mechanisms in flutter-vibration studied in unanesthetized monkeys. Neuronal periodicity and frequency discrimination. J. Neurophysiol. 32, 452484. doi: $10.1152 /$ jn.1969.32.3.452

O’Doherty, J. E., Lebedev, M. A., Ifft, P. J., Zhuang, K. Z., Shokur, S., Bleuler, H., et al. (2011). Active tactile exploration using a brain-machine-brain interface. Nature 479, 228-231. doi: 10.1038/nature10489

O’Doherty, J. E., Lebedev, M. A., Li, Z., and Nicolelis, M. A. (2012). Virtual active touch using randomly patterned intracortical microstimulation. IEEE Trans. Neural. Syst. Rehabil. Eng. 20, 85-93. doi: 10.1109/TNSRE.2011.2166807

Overstreet, C. K., Hellman, R. B., Ponce Wong, R. D., Santos, V. J., and Helms Tillery, S. I. (2016). Discriminability of single and multichannel intracortical microstimulation within somatosensory cortex. Front. Bioeng. Biotechnol. 4:91. doi: 10.3389/fbioe.2016.00091

Raspopovic, S., Capogrosso, M., Petrini, F. M., Bonizzato, M., Rigosa, J., Di Pino, G., et al. (2014). Restoring natural sensory feedback in real-time bidirectional hand prostheses. Sci. Transl. Med. 6:222ra19. doi: 10.1126/scitranslmed. 3006820

Romo, R., Hernandez, A., Zainos, A., Brody, C. D., and Lemus, L. (2000). Sensing without touching: psychophysical performance based on cortical microstimulation. Neuron 26, 273-278. doi: 10.1016/s0896-6273(00)81156-3

Romo, R., Hernandez, A., Zainos, A., and Salinas, E. (1998). Somatosensory discrimination based on cortical microstimulation. Nature 392, 387-390. doi: $10.1038 / 32891$

Salinas, E., Hernandez, A., Zainos, A., and Romo, R. (2000). Periodicity and firing rate as candidate neural codes for the frequency of vibrotactile stimuli. J. Neurosci. 20, 5503-5515. doi: 10.1523/jneurosci.20-14-05503.2000

Suminski, A. J., Tkach, D. C., Fagg, A. H., and Hatsopoulos, N. G. (2010). Incorporating feedback from multiple sensory modalities enhances brainmachine interface control. J. Neurosci. 30, 16777-16787. doi: 10.1523/ JNEUROSCI.3967-10.2010

Tabot, G. A., Dammann, J. F., Berg, J. A., Tenore, F. V., Boback, J. L., Vogelstein, R. J., et al. (2013). Restoring the sense of touch with a prosthetic hand through a brain interface. Proc. Natl. Acad. Sci. U.S.A. 110, 18279-18284. doi: 10.1073/ pnas. 1221113110

Tabot, G. A., Kim, S. S., Winberry, J. E., and Bensmaia, S. J. (2015). Restoring tactile and proprioceptive sensation through a brain interface. Neurobiol. Dis. 83, 191-198. doi: 10.1016/j.nbd.2014.08.029

Tan, D. W., Schiefer, M. A., Keith, M. W., Anderson, J. R., Tyler, J., and Tyler, D. J. (2014). A neural interface provides long-term stable natural touch perception. Sci. Transl. Med. 6:257ra138. doi: 10.1126/scitranslmed.3008669

Vardar, B., and Guclu, B. (2017). Non-NMDA receptor-mediated vibrotactile responses of neurons from the hindpaw representation in the rat SI cortex. Somatosens. Mot. Res. 34, 189-203. doi: 10.1080/08990220.2017.1390450

Vidal, G. W., Rynes, M. L., Kelliher, Z., and Goodwin, S. J. (2016). Review of brain-machine interfaces used in neural prosthetics with new perspective on somatosensory feedback through method of signal breakdown. Scientifica 2016:8956432. doi: 10.1155/2016/8956432

Conflict of Interest Statement: The authors declare that the research was conducted in the absence of any commercial or financial relationships that could be construed as a potential conflict of interest.

Copyright (®) 2019 Kramer, Lamorie-Foote, Barbaro, Lee, Peng, Gogia, Liu, Kellis and Lee. This is an open-access article distributed under the terms of the Creative Commons Attribution License (CC BY). The use, distribution or reproduction in other forums is permitted, provided the original author(s) and the copyright owner(s) are credited and that the original publication in this journal is cited, in accordance with accepted academic practice. No use, distribution or reproduction is permitted which does not comply with these terms. 